\title{
Domain Decomposition Methods for Time-Harmonic Electromagnetic Waves with High Order Whitney Forms
}

\author{
Nicolas Marsic ${ }^{1}$, Caleb Waltz ${ }^{2}$ Jin-Fa Lee ${ }^{2}$, Christophe Geuzaine $^{1}$ \\ ${ }^{1}$ Department of Electrical Engineering and Computer Science, University of Liège, Belgium \\ ${ }^{2}$ ElectroScience Laboratory, The Ohio State University, Columbus, OH 43212, USA
}

\begin{abstract}
Classically, domain decomposition methods (DDM) for time-harmonic electromagnetic wave propagation problems make use of the standard, low order, Nédélec basis functions. This paper analyzes the convergence rate of DDM when higher order finite elements are used for both volume and interface discretizations, in particular when different orders are used in the volume and on the interfaces.
\end{abstract}

Index Terms-Computational electromagnetics, Domain decomposition, Finite element analysis, High performance computing.

\section{INTRODUCTION}

$\mathbf{T}$ HERE is a growing consensus that state of the art finite element technology requires, and will continue to require, too extensive computational resources to provide the necessary resolution for complex high frequency electromagnetic simulations, even at the rate of computational power increase. This leads us to consider methods with a higher order of grid convergence than the classical second order provided by most industrial grade codes.

Moreover, the direct application of the finite element method (FEM) on these high frequency problems leads to very large, complex and possibly indefinite linear systems. Unfortunately, direct sparse solvers do not scale well for solving such large systems, and Krylov subspace iterative solvers can exhibit slow convergence or even diverge [1]. Domain decomposition methods (DDM) provide an elegant alternative, iterating between sub-problems of smaller sizes, amenable to sparse direct solvers [2].

In this paper we investigate the use of high order Whitney forms for the discretization of the sub-problems as well as the interface conditions between the sub-domains.

\section{Problem Definition}

Let us start by considering the time-harmonic propagation of an electrical wave $\mathbf{e}$ in an open waveguide $\Omega$ with metallic boundaries $\Gamma^{0}$. A source signal $\mathrm{e}^{s}$ is imposed on $\Gamma^{s}$. In order to solve this problem with the FEM, the infinite domain is truncated by a fictitious boundary $\Gamma^{\infty}$, on which a SilverMüller radiation condition is used. This leads to the following problem:

$$
\left\{\begin{aligned}
\text { curl curl e }-k^{2} \mathbf{e} & =\mathbf{0} & & \text { on } \Omega, \\
\gamma_{\mathrm{T}}(\mathbf{e}) & =\mathbf{0} & & \text { on } \Gamma^{0}, \\
\gamma_{\mathrm{T}}(\mathbf{e}) & =\mathbf{e}^{s} & & \text { on } \Gamma^{s}, \\
\gamma_{\mathrm{t}}(\operatorname{curl} \mathbf{e})+\jmath k \gamma_{\mathrm{T}}(\mathbf{e}) & =\mathbf{0} & & \text { on } \Gamma^{\infty},
\end{aligned}\right.
$$

Manuscript received April 1, 2015; revised May 15, 2015 and June 1, 2015; accepted July 1, 2015. Date of publication July 10, 2015; date of current version July 31, 2015. Corresponding author: N. Marsic (e-mail: nicolas.marsic@ulg.ac.be). Color versions of one or more of the figures in this paper are available online at http://ieeexplore.ieee.org. Digital Object Identifier (inserted by IEEE). where $k$ is the wavenumber, $\jmath$ the imaginary unit, and the tangential trace and tangential component trace operators are given by $\gamma_{\mathrm{T}}(\mathbf{v}): \mathbf{v} \mapsto \mathbf{n} \times \mathbf{v} \times \mathbf{n}$ and $\gamma_{\mathrm{t}}(\mathbf{v}): \mathbf{v} \mapsto \mathbf{n} \times \mathbf{v}$, with $\mathbf{n}$ as the unit vector outwardly oriented normal to $\Omega$.

\section{NON-OVERLAPPING DDM}

Let us now review the construction of a non-overlapping additive Schwarz domain decomposition method for the propagation problem (1).

We start by decomposing the domain $\Omega$ into nonoverlapping sub-domains $\Omega_{i}$, with $i \in\left\{1, \ldots, N_{d o m}\right\}$. On a given sub-domain $\Omega_{i}$, the interface with sub-domain $\Omega_{j}$ is denoted by $\Sigma_{i j}$. Conversely, on sub-domain $\Omega_{j}$, the interface with sub-domain $\Omega_{i}$ is written $\Sigma_{j i}$. The electric field on $\Omega_{i}$ is denoted by $\mathbf{e}_{i}$.

It can be shown [2] that the solution e of [1], on the whole domain $\Omega$, can be computed by the following iterative scheme (indexed by $p$ ):

$$
\left\{\begin{aligned}
\operatorname{curl} \operatorname{curl} \mathbf{e}_{i}^{p}-k^{2} \mathbf{e}_{i}^{p} & =\mathbf{0} & & \text { on } \Omega_{i}, \\
\gamma_{\mathrm{T}}\left(\mathbf{e}_{i}^{p}\right) & =\mathbf{0} & & \text { on } \Gamma_{i}^{0}, \\
\gamma_{\mathrm{T}}\left(\mathbf{e}_{i}^{p}\right) & =\mathbf{e}^{s} & & \text { on } \Gamma_{i}^{s}, \\
\gamma_{\mathrm{t}}\left(\operatorname{curl} \mathbf{e}_{i}^{p}\right)+\jmath k \gamma_{\mathrm{T}}\left(\mathbf{e}_{i}^{p}\right) & =\mathbf{0} & & \text { on } \Gamma_{i}^{\infty}, \\
\gamma_{\mathrm{t}}\left(\operatorname{curl} \mathbf{e}_{i}^{p}\right)+\mathcal{S}\left[\gamma_{\mathrm{T}}\left(\mathbf{e}_{i}^{p}\right)\right] & =\mathbf{g}_{i j}^{p-1} & & \text { on } \Sigma_{i j},
\end{aligned}\right.
$$

with

$$
\mathbf{g}_{i j}^{p}=-\mathbf{g}_{j i}^{p-1}+2 \mathcal{S}\left[\gamma_{\mathrm{T}}\left(\mathbf{e}_{j}^{p}\right)\right] \quad \text { on } \Sigma_{i j} .
$$

The quantity $\mathbf{g}_{i j}^{p}$ represents the coupling of $\Omega_{i}$ with $\Omega_{j}$, and the operator $\mathcal{S}$ is a well chosen boundary transmission condition. A short presentation of optimized boundary conditions can be found in [3]. Let us remark that, in its simplest form (zeroth order), the $\mathcal{S}$ operator is simply a complex value: $\mathcal{S}=\jmath k$.

It is worth noticing [3] that solving iteratively (2) and (3) can be rewritten as the application of the iteration operator $\mathcal{A}$ :

$$
\mathbf{g}^{p}=\mathcal{A} \mathbf{g}^{p-1}+\mathbf{b},
$$

where $\mathbf{g}^{p}$ is the concatenation of the $\mathbf{g}_{i j}^{p}$ for $1 \leq i, j \leq N_{d o m}$, and $\mathbf{b}$ contains the contribution of the source electric field. Thus (2) and (3) can be solved using a Krylov solver applied to:

$$
(\mathcal{I}-\mathcal{A}) \mathbf{g}=\mathbf{b},
$$


where $\mathcal{I}$ is the identity operator. The set of sub-problems in (2) can be solved independently and are of relatively small size, since they are defined on small sub-domains. This property allows us to use (sparse) direct solvers. It is worth noticing that the interface problem is solved using a matrix-free iterative solver. Thus, the operator $(\mathcal{I}-\mathcal{A})$ is never explicitly constructed: only its application is required.

\section{High ORder Discretizations}

Classically, DDM implementations make use of the standard Nédélec basis functions [4]. Here we analyze the behavior of the DDM when higher order bases are used, which are paramount to the accurate solution of high frequency propagation problems, thanks to their improved dispersion properties [5]. In particular, we investigate the use of the high order hierarchical Whitney forms on tetrahedra proposed in [6], which associate the higher order degrees of freedom to the edges, faces and volumes of the mesh elements. The hierarchical nature of the bases allows to easily mix elements of different orders in the same mesh. An efficient thread-based parallel assembler [7] is used to mitigate the drawback of such higher order elements, that is the high assembly time of the FEM matrix.

In order to analyze both the accuracy and the efficiency of the DDM with high order discretizations, we vary the FEM discretization order of both $\mathbf{e}_{i}$ and $\mathbf{g}$, with basis orders ranging between 1 and 4 . (Order 1 refers to the complete first order basis, not the standard Nédélec basis). We also vary the mesh density from 1 to 32 elements per wavelength. Since multiple basis orders are considered, the following notation is used: $O\{e, g\}$, where $e$ is the order used for $\mathbf{e}_{i}$ and $g$ the order for g. We use the term non-mixed DDM when the same basis orders are employed, and mixed DDM in the opposite case.

From the DDM point for view, we consider the following transmission conditions: $i$ ) zeroth order [2]; ii) optimized second order [8]; iii) Padé-localized square-root [9]. It is worth noticing that higher order transmission conditions require auxiliary unknowns. Based on empirical results, the best performance was found when those unknowns are discretized using order $g$ for the optimized second order conditions, and using order $e$ for the Padé-localized square-root conditions.

\section{NUMERICAL EXPERIMENTS}

We consider the propagation problem (1) in a rectangular waveguide 1.7 wavelengths long, with two sub-domains, where the source field excites the $\mathrm{TM}_{1,1}$ mode at $1 \mathrm{GHz}$. The linear system (4) is solved using a non-restarted GMRES with a relative tolerance set to $10^{-9}$ (from the PETSc [10] library). The sub-problems (2) are solved with the direct sparse solver MUMPS [11].

\section{A. Solution accuracy}

Let us start by analyzing the accuracy of the solution with respect to the mesh size and the FEM discretization order, using the zeroth transmission condition. Fig. 1 presents the $L_{2}$ error between the analytic solution [12] and a given simulation, and Table [1] summarizes the measured convergence rates. The optimal convergence rate $\mathcal{O}\left(h^{p+1}\right)$ (where $p$ is the polynomial order used and $h$ the mesh elements size [13]) is recovered for sufficiently fine meshes, which validates the implementation. Solving the full problem in (17) or the DDM problem in (2) and $(3)$ is equivalent up to the iterative solver tolerance. It is then worth motioning that, for the non-mixed cases, the same solution accuracy was recorded when no DDM was used.

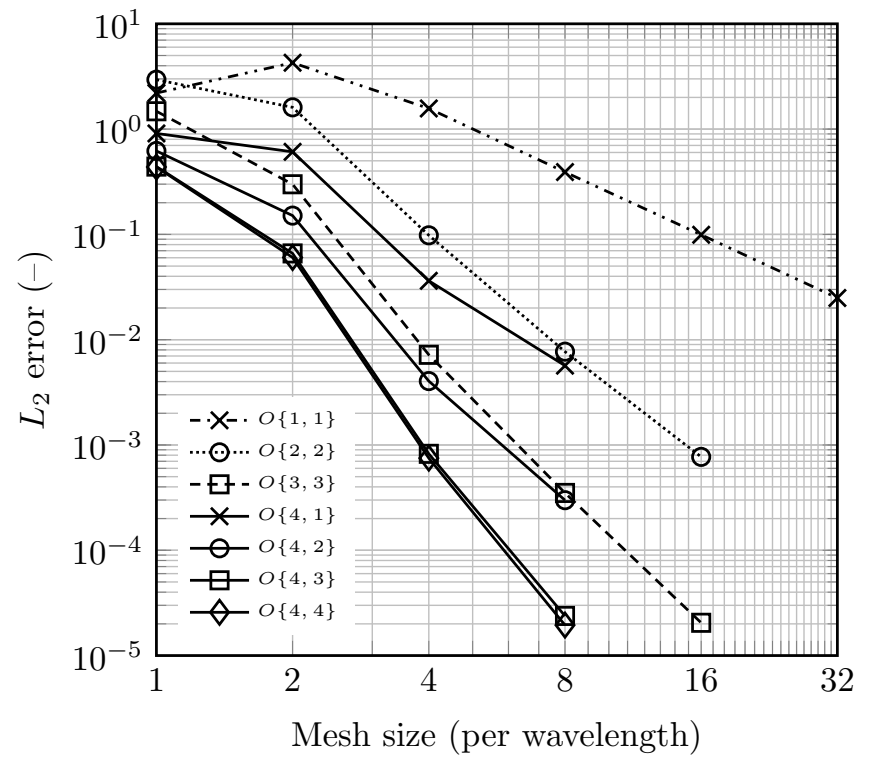

Fig. 1. Relative error between analytic and FEM solution for different discretization orders, when a zeroth order transmission condition is used.

TABLE I

CONVERGENCE RATE FOR NON-MIXED DDM.

\begin{tabular}{|c|c|c|}
\hline FEM Order & Measured convergence rate & Optimal convergence rate \\
\hline 1 & 2.0 & 2 \\
\hline 2 & 3.3 & 3 \\
\hline 3 & 4.1 & 4 \\
\hline 4 & 5.3 & 5 \\
\hline
\end{tabular}

A decrease in the solution accuracy is observed on Fig. 11 for mixed-order cases, which can be explained by the low-pass filtering introduced by the sub-discretization of $\mathbf{g}$ with respect to $\mathbf{e}$ in the last equation of (2). In this situation, equation (1) is no longer equivalent to equations (2) and (3) at the discrete level. For each mixed-order, the relative error with respect to the analytic solution is reported on Table III For conciseness, only the 8 mesh elements per wavelength case is considered. If the error with respect to the non-mixed case is increased, it still remains in an acceptable range.

The behavior of the optimized second order and the Padélocalized transmissions conditions is fairly similar to the zeroth order condition. For non-mixed orders, using higher order transmission conditions does not change the solution accuracy compared to the original problem (e.g., every order $O\{4,4\}$ in Table [I leads to the same accuracy). In the case of mixed orders, for the two high order conditions, the solution accuracy is reduced compared to the zeroth order one. However, as for the zeroth order case, the solution error remains in an acceptable range (see Table II). 
TABLE II

RELATIVE ERRORS AND ITERATION COUNTS FOR MIXED ORDERS SIMULATIONS.

\begin{tabular}{|c|c|c|c|c|}
\hline Condition & Mesh size & Order & Error & Iteration count \\
\hline \multirow{4}{*}{ Zeroth } & \multirow{4}{*}{8} & $O\{4,1\}$ & $5.7 \times 10^{-3}$ & 71 \\
\hline & & $O\{4,2\}$ & $2.9 \times 10^{-4}$ & 76 \\
\hline & & $O\{4,3\}$ & $2.4 \times 10^{-5}$ & 81 \\
\hline & & $O\{4,4\}$ & $1.9 \times 10^{-5}$ & 115 \\
\hline \multirow{4}{*}{ Second } & \multirow{4}{*}{8} & $O\{4,1\}$ & $3.9 \times 10^{-2}$ & 62 \\
\hline & & $O\{4,2\}$ & $2.7 \times 10^{-3}$ & 53 \\
\hline & & $O\{4,3\}$ & $1.3 \times 10^{-4}$ & 44 \\
\hline & & $O\{4,4\}$ & $1.9 \times 10^{-5}$ & 39 \\
\hline \multirow{4}{*}{ Padé } & \multirow{4}{*}{8} & $O\{4,1\}$ & $8.4 \times 10^{-3}$ & 15 \\
\hline & & $O\{4,2\}$ & $1.7 \times 10^{-4}$ & 15 \\
\hline & & $O\{4,3\}$ & $7.2 \times 10^{-5}$ & 22 \\
\hline & & $O\{4,4\}$ & $1.9 \times 10^{-5}$ & 31 \\
\hline
\end{tabular}

\section{B. Iteration count}

Let us now study the iteration count of the DDM iterative solver. Fig. 2 depicts the DDM iteration count for different basis orders and transmission conditions. To complete this data set, Table II reports some numerical values for a few mixed order cases.

For the zeroth order and the Padé-localized square-root conditions, mixed orders lead to a substantial decrease in the iteration count. On the other hand, mixed orders tend to increase the iteration count for the optimized second order condition. In the case of non-mixed orders, for the zeroth order condition, we can notice a significant iteration count increase when high order discretizations are used. On the other hand, for the higher order transmission conditions, the discretization order increase doesn't seems to impact significantly the iteration count.

Alone, this last analysis is not sufficient to assess the performance of high order DDM. Indeed, the problems solved for the different test cases do not represent exactly the same phenomenon, since different discretization orders are used. In order to have a better comparison, let us now consider simulations leading to an accuracy of same magnitude, as shown on Table III. It is worth recalling that mixed order discretizations lead to different errors, depending on the transmission condition used. Thus, the iteration count cannot be reported for the three conditions with the exact same accuracy. Unavailable data are recorded by a dash.

For non-mixed simulations we observe that the iteration counts, for a given transmission condition and accuracy, are not significantly impacted by discretization order and mesh size modifications. It is worth recalling that, in a first order discretization context, the two higher order transmission conditions are known to be robust with respect to the mesh refinement. For the mixed order cases, as mentioned previously, the iteration count is substantially reduced, except for the optimized second order case.

\section{ILLUSTRATIVE EXAMPLE}

Let us now consider a less academic example: an open segmented waveguide for photonics applications [14].

Basically, this waveguide consists in a chain of several equispaced non-metallic cylinders in open space. For some

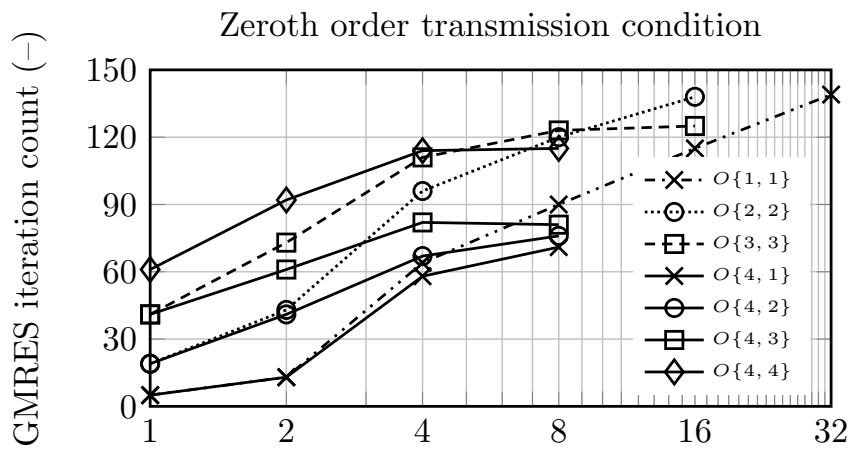

Optimized second order transmission condition

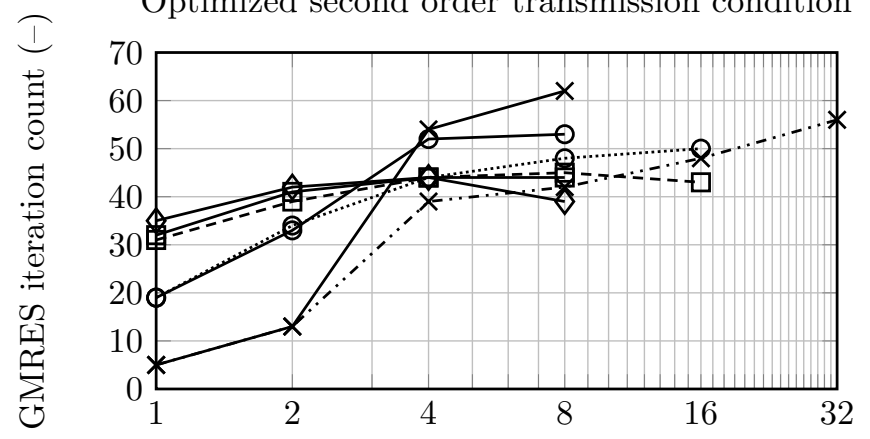

Padé-localized square-root transmission condition

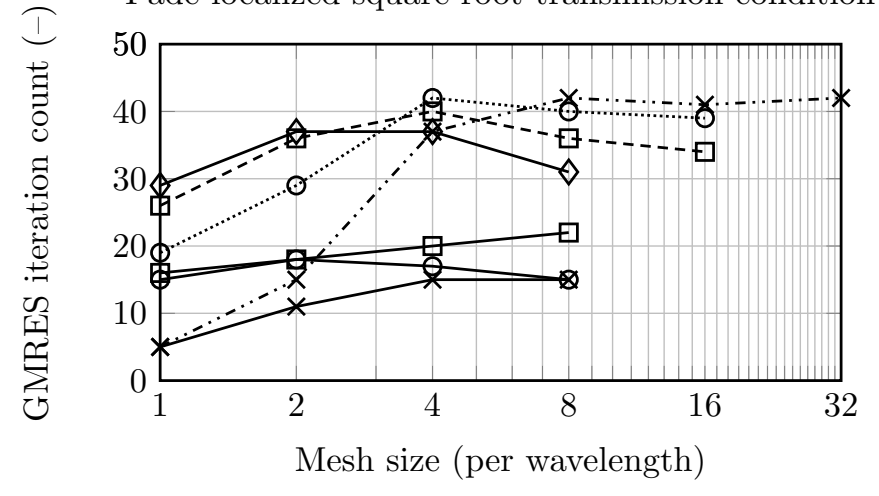

Fig. 2. DDM iteration count for different discretization orders.

frequencies, the interference pattern between the cylinders may lead to a guided wave, even if the system is open. Because of the cylindrical nature of the geometry, curved mesh elements are a natural choice. However, using this family of geometrical elements require the use of high order discretization at the FEM level.

Preliminary results are shown on Table IV It is worth noticing that the high iteration count is explained by the lack of preconditioning of the iterative solver [15]. Without it, the iteration count increases with the number of sub-domains. The Padé-localized transmission condition was used on 24 subdomains with 10 order 2 mesh elements per wavelength. The number of unknowns and the memory consumption are given as the mean and standard deviation per sub-problems. Fig. 3 depicts the real part of the $z$-component (i.e., along the rods) of $\mathbf{e}_{i}$.

As shown on Table [V] a ten million three dimensional electromagnetic problem was simulated in a few hours with a 
TABLE III

COMPARISONS OF SIMULATIONS LEADING TO THE SAME ACCURACY.

\begin{tabular}{|c|c|c|c|c|c|}
\hline \multirow[b]{2}{*}{ Order } & \multirow[b]{2}{*}{ Mesh size } & \multirow[b]{2}{*}{ Accuracy } & \multicolumn{3}{|c|}{ Iteration count } \\
\hline & & & Zeroth & Second & Padé \\
\hline$O\{1,1\}$ & 16 & $9.9 \times 10^{-2}$ & 115 & 48 & 41 \\
\hline$O\{2,2\}$ & 4 & $9.8 \times 10^{-2}$ & 96 & 44 & 42 \\
\hline$O\{4,3\}$ & 2 & $6.6 \times 10^{-2}$ & 61 & - & - \\
\hline$O\{4,3\}$ & 2 & $7.3 \times 10^{-2}$ & - & 41 & - \\
\hline$O\{4,3\}$ & 2 & $6.7 \times 10^{-2}$ & - & - & 18 \\
\hline$O\{2,2\}$ & 16 & $7.7 \times 10^{-4}$ & 138 & 50 & 39 \\
\hline$O\{4,4\}$ & 4 & $7.5 \times 10^{-4}$ & 114 & 44 & 37 \\
\hline$O\{4,3\}$ & 4 & $8.2 \times 10^{-4}$ & 82 & - & - \\
\hline$O\{4,3\}$ & 4 & $1.6 \times 10^{-3}$ & - & 44 & - \\
\hline$O\{4,3\}$ & 4 & $1.1 \times 10^{-3}$ & - & - & 20 \\
\hline$O\{3,3\}$ & 16 & $2.1 \times 10^{-5}$ & 125 & 43 & 34 \\
\hline$O\{4,4\}$ & 8 & $1.9 \times 10^{-5}$ & 115 & 39 & 31 \\
\hline
\end{tabular}

TABLE IV

Preliminary RESUlts on A SEgMENTED WAVEguide.

\begin{tabular}{|c|c|c|c|c|}
\hline Order & Iteration count & Memory & Unknowns $\mathbf{e}_{i}$ & Time \\
\hline$O\{3,2\}$ & 297 & $17 \pm 2(\mathrm{~GB})$ & $422 \mathrm{k} \pm 31 \mathrm{k}$ & $2(\mathrm{~h})$ \\
\hline$O\{3,3\}$ & 410 & $18 \pm 2(\mathrm{~GB})$ & $422 \mathrm{k} \pm 31 \mathrm{k}$ & $4(\mathrm{~h})$ \\
\hline
\end{tabular}

high order FEM discretization. The high precision simulation was prepared using mixed orders, thus enabling fast tests with an acceptable accuracy. Finally, it is worth noticing the excellent memory distribution across the computing nodes, which is usually a serious limitation of direct solvers.

\section{CONCLUSIONS}

In this paper, we analyzed, using numerical experiments, the performance of different optimized domain decomposition methods when used with high order FEM discretizations, in terms of both solution accuracy and iteration count.

Two situations where considered for discretizing the physical unknowns $\mathbf{e}_{i}$ and the interface unknowns $\left.\mathbf{g :} i\right)$ the same basis order is used (non-mixed order); $i$ ) different basis orders are used (mixed orders). We showed that, when the solution accuracy is held constant, the DDM iteration count is not significantly impacted by mesh size and discretization order changes. We also showed that using mixed orders leads to a significant iteration count improvement, but at an accuracy decrease cost. These mixed orders are then suitable for preliminary simulations with acceptable accuracy.

Overall, the combination of the optimized DDM (using a process-based parallelism) with new efficient high-order assembly methods (using a thread-based parallelism) leads to a very precise, efficient and flexible simulation tool, suitable for solving (very) large electromagnetic problems on high performance heterogeneous computation platforms.

\section{ACKNOWLEDGMENT}

N. Marsic is a fellowship beneficiary with the Belgian Research Training Fund for Industry and Agriculture (FRIA). This work was also supported in part by the Belgian Science Policy Office under grant IAP P7/02. Computational resources

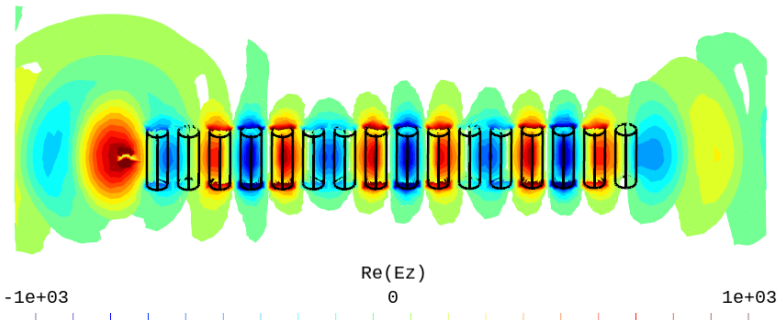

Fig. 3. Segmented waveguide: real part of the electrical field $z$-component.

have been provided by CÉCI, funded by F.R.S.-FNRS (Fonds de la Recherche Scientifique) under grant $\mathrm{n}^{\circ} 2.5020 .11$, and the Tier-1 supercomputer of the Fédération Wallonie-Bruxelles, funded by the Walloon Region under grant $n^{\circ} 1117545$.

\section{REFERENCES}

[1] O. G. Ernst and M. J. Gander, "Why it is difficult to solve Helmholtz problems with classical iterative methods," in Numerical Analysis of Multiscale Problems (I. G. Graham, T. Y. Hou, O. Lakkis, and R. Scheichl, eds.), vol. 83 of Lecture Notes in Computational Science and Engineering, pp. 325-363, Springer Berlin Heidelberg, 2012.

[2] B. Després, P. Joly, and J. E. Roberts, "A domain decomposition method for the harmonic Maxwell equations," Iterative methods in linear algebra (Brussels, 1991), pp. 475-484, 1992.

[3] C. Geuzaine, B. Thierry, N. Marsic, D. Colignon, A. Vion, S. Tournier, Y. Boubendir, M. El Bouajaji, and X. Antoine, "An open source domain decomposition solver for time-harmonic electromagnetic wave problems," in Antenna Measurements Applications (CAMA), 2014 IEEE Conference on, pp. 1-4, 2014.

[4] J.-C. Nédélec, "Mixed finite elements in $R^{3}$," Numerische Mathematik, vol. 35, pp. 315-341, 1980.

[5] F. Ihlenburg and I. Babuška, "Finite element solution of the Helmholtz equation with high wave number part II: The h-p version of the FEM," SIAM Journal on Numerical Analysis, vol. 34, no. 1, pp. 315-358, 1997.

[6] S. Zaglmayr, "High order finite element methods for electromagnetic field computation," $\mathrm{PhD}$ thesis, Johannes Kepler Universität, Austria, 2006.

[7] N. Marsic and C. Geuzaine, "Efficient finite element assembly of high order Whitney forms," IET Science, Measurement \& Technology, vol. 9, pp. 204-210, 2015.

[8] V. Rawat and J.-F. Lee, "Nonoverlapping domain decomposition with second order transmission condition for the time-harmonic Maxwell's equations," SIAM Journal on Scientific Computing, vol. 32, no. 6, pp. 3584-3603, 2010.

[9] M. El Bouajaji, B. Thierry, X. Antoine, and C. Geuzaine, "A quasi-optimal domain decomposition algorithm for the time-harmonic Maxwell's equations," Journal of Computational Physics, vol. 294, no. 1, pp. $38-57,2015$.

[10] S. Balay, S. Abhyankar, M. F. Adams, J. Brown, P. Brune, K. Buschelman, V. Eijkhout, W. D. Gropp, D. Kaushik, M. G. Knepley, L. C. McInnes, K. Rupp, B. F. Smith, and H. Zhang, "PETSc users manual," Tech. Rep. ANL-95/11 - Revision 3.5, Argonne National Laboratory, 2014.

[11] P. R. Amestoy, I. S. Duff, J. Koster, and J.-Y. L'Excellent, "A fully asynchronous multifrontal solver using distributed dynamic scheduling," SIAM Journal on Matrix Analysis and Applications, vol. 23, no. 1, pp. 15-41, 2001.

[12] L. C. Shen and J. A. Kong, Applied Electromagnetism. PWS Publishing Company, 1995.

[13] F. Ihlenburg and I. Babuška, "Finite element solution of the Helmholtz equation with high wave number part I: The h-version of the FEM," Computers \& Mathematics with Applications, vol. 30, no. 9, pp. 9 - 37, 1995.

[14] A. Nicolet, G. Demesy, F. Zolla, and B. Vial, "Quasi-modal analysis of segmented waveguides," in Antenna Measurements Applications (CAMA), 2014 IEEE Conference on, pp. 1-4, 2014.

[15] A. Vion and C. Geuzaine, "Double sweep preconditioner for optimized schwarz methods applied to the helmholtz problem," Journal of Computational Physics, vol. 266, pp. 171 - 190, 2014 